\title{
A Preliminary Study of the Assessment of Visual Space Recognition in Stroke Patients using New Head Mounted Display (HMD) System with Visual Image Transformation.
}

\author{
Toshiaki Tanaka, Syunichi Sugihara, Norio Kato, Tomoya Miyasaka, Takashi Izumi
}

\begin{abstract}
Background> Various disturbances of visual space recognition include unilateral spatial neglect (USN) and visual field defects (VFD) has been strongly associated with an increased risk for injury and with poor functional outcome. To quantitatively evaluate visual-spatial recognition in cerebrovascular disease patients, we developed a new measurement system able to present a versatile visual information. The system uses a head-mounted display (HMD) as the device that presents visual information. The purpose of this study was the development of a method for presenting diverse visual information and assessing visual space recognition using a new head mounted display (HMD) system. <Methods> A total of eight subjects participated in this study, comprising four with USN and four with VFD. A test sheet was placed on a desk, and its image was projected on the display of the HMD. Then, space recognition assessment was conducted using a cancellation test and motion analysis of the eye, head and trunk movements under four conditions with images reduced in size and shifted. <Results> Leftward visual search was dominant in VFD patients, while rightward visual search was dominant in USN patients. The angular velocity of leftward eye movement during visual search of the right sheet decreased in both patient types. Motion analysis revealed a tendency of VFD patients to rotate the head in the affected direction under the left reduction condition, whereas USN patients rotated it in the opposite direction of the neglect. <Conclusion> A new HMD system was developed for presenting diverse visual information and assessing visual space recognition which identified the differences in the disturbance of visual space recognition of VFD and USN patients were indicated. The HMD system may be available and effective for evaluating and training visual recognition and ADLs in VFD and USN patients as a clinical device.
\end{abstract}

Index Terms - Unilateral spatial neglect, Visual field defects, head-mounted display, Visual space recognition, virtual reality.

Toshiaki Tanaka, Institute of Gerontology, The University of Tokyo, Tokyo, Japan, Centre d'études avancées franco-japonais de Paris, pairs, France., Department of Physical Therapy, Faculty of Health Sciences, Hokkaido University of Science, Sapporo, Hokkaido, Japan

Syunichi Sugihara, Department of Rehabilitation, Sapporo Shuyukai Hospital, Sapporo, Hokkaido, Japan

Norio Kato, Department of Physical Therapy, Faculty of Health Sciences, Hokkaido University of Science, Sapporo, Hokkaido, Japan

Tomoya Miyasaka, Department of Physical Therapy, Faculty of Health Sciences, Hokkaido University of Science, Sapporo, Hokkaido, Japan

Takashi Izumi, Department of Human Science and Informatics, School of Biological Science and Engineering, Tokai University, Kumamoto, Japan

\section{INTRODUCTION}

The problems of visual recognition have been strongly associated with an increased risk for injury and with poor activities of daily living (ADL). Typical disturbances of visual space recognition include unilateral spatial neglect (USN) and visual field defects (VFD). However, many aspects of these disorders remain unknown, such as their differences. It is believed that VFD is present in about $40 \%$ of patients with cerebrovascular diseases, and that it is spontaneously recovered in $50-60 \%$ of them [1]. Principal lesion locations include the lateral geniculate body and temporal lobe [2], and occipital lobe [3], where homonymous hemianopsia reportedly occurs. A recent report has described the efficacy of rehabilitation for VFD [4], indicating the efficacy of the compensation method by eye movement. A trend of capturing a visual object in the central visual field to acquire accurate information has also been described [5]. VFD patients try to obtain visual information by concentrating on a field area that remains in the central visual field. Accordingly, it is necessary for them to acquire information by eye movement to capture an object in an unaffected visual field and head rotation toward the affected visual field. These actions are usually designated as visual search.

Several assessment methods of visual search reportedly exist, and include a method of measuring the search range for a designated target with the head fixed, and a method of ascertaining the search time and the number of correct answers in a search for a geometric configuration [6,7]. Moreover, accurate comprehension of pathology requires modification of a head movement state and the range of instruction without body constraint as in ADL. However, few reports have described the measurement or analysis of eye movement at the time of presenting such diverse visual information.

Disturbances of space recognition following USN include disorders such as colliding with people or objects during activities of daily living or wheelchair accidents caused by failure to operate the brake and footrest on the side of neglect field. Accordingly, supervision by a caregiver is often indispensable, even after recovery of motor function by rehabilitation, so USN is a great hindrance to the independence of people with disability [8]. The onset of USN is mostly observed in patients with cerebrovascular diseases 
in the right hemisphere from the acute stage to chronic stages [9]. It is less frequent in patients with cerebrovascular diseases in the left hemisphere, persisting only slightly until the chronic stage [10]. Test methods for USN include a cancellation test, copy test, drawing test, and line bisection test. The behavioral inattention test (BIT) normalizes these, including laboratory tests imitating daily living scenes [11]. The Catherine Bergego scale (CBS) is an assessment method for unilateral spatial neglect symptoms in ADL, for which therapists evaluate activities of 10 types in a real situation by observation. USN is observed even in cases where USN is not observed by desk checking according to CBS [12], so the CBS is regarded as useful as a USN assessment method in ADL.

Hillis revealed differences in focal sites due to neglect related to the deviation of egocentric and allocentric standards using magnetic resonance imaging (MRI) [13, 14]. Consequently, it is commonly considered that methods for recognizing exterior space include a reference frame centering on an individual's own body (egocentric reference frame) and that centering on an object (allocentric reference frame). USN is classified roughly into the following two types: egocentric neglect, in which the viewer-centered unilateral space is neglected centering on the truncus, head, and retina; and allocentric neglect, in which the unilateral space of an object is neglected centering on the object itself in the exterior space, irrespective of the relation to the individual's own body position. Patients with unilateral neglect may be influenced by both or either egocentric or allocentric deficiency, but little is known about the neurology and pathology underlying the different frames of reference.

Virtual reality (VR) has many advantages over other ADL rehabilitation techniques and offers the potential to enhance a human performance testing and training environment [15]. VR has been investigated in a few studies using devices for augmentation of visual information. For example, there is one approach where head mounted display (HMD) gives a patient with Parkinson' disease an emphasized visual input in order to improve a frozen gait of the patient [16]. HMD has a function which can focus on a certain object or to limit the surrounding environmental conditions, and to offer versatile visual information. Therefore, HMD can produce both egocentric and allocentric conditions for evaluation of a USN patient.

Our previous studies analyzed an evaluation process system of USN in various visual fields using HMD in order to understand more accurately any faults of USN operating in the object-centred coordinates [17, 18]. The results showed that the assessment of USN using an HMD system may clarify the left neglected area which cannot be easily observed in the clinical evaluation for USN in the object-centred co-ordinates. Moreover, USN symptoms not disclosed by the ordinary line cancellation test were found by the HMD system. Eyes and head movement analysis were also added to our HMD system [19, 20]. Various reports have described the assessment of the eye movement of left USN patients [21-23]. However, few studies have analyzed impairment of the visual field in VFD and USN without body constraint in relation to ADL. The differences between the visual problems of VFD and USN have not been clarified under diverse visual conditions during the simultaneous measurement of movement of the eye, head and trunk. Therefore, the purpose of this study was to assess

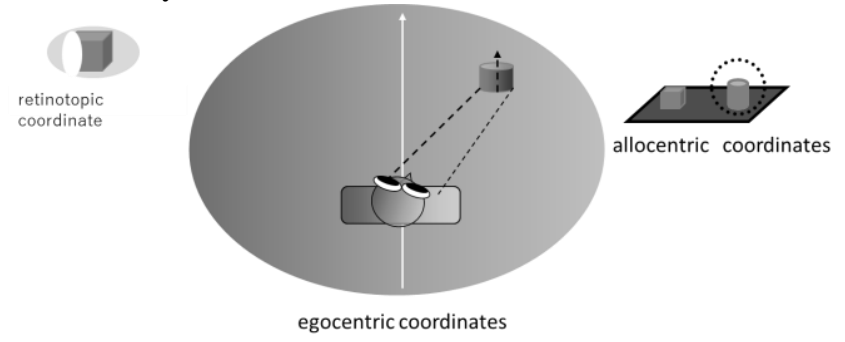

Task-dependent differences in the Visual search?

Figure. 1 The analysis of USN space coordinate system theory

the eye movements of USN and VFD patients and to clarify the characteristics of the disturbance of spatial recognition.

\section{Methods}

\section{A. A novel assessment system using a head-mounted display}

\section{1) Space coordinate system theory}

Visual input enabling perception of the external world has been proposed to be divisible into viewer-based (egocentric) and object-based (allocentric) coordinate systems (Fig. 1). In the egocentric coordinate system, the direction and position of spatial targets on the coordinate system change as the viewer moves through space. This system comprises multiple operational parts, such as the eyes, head, and trunk, which become frames of reference for differential encoding of the position and direction of spatial targets on the coordinate system. Unilateral spatial neglect (USN) can therefore be divided into two categories. In egocentric neglect, the trunk, head, or retinas of the viewer determine which hemispace is neglected. On the other hand, in allocentric (object-based) neglect, the target object in the external world becomes the basis for the neglected hemispace regardless of the position of the body [24-27].

According to perfusion-weighted magnetic resonance imaging (MRI) studies, patients with allocentric neglect exhibit low perfusion in the superior temporal gyrus, while those with egocentric neglect exhibit low perfusion around the angular gyrus, particularly in the inferior frontal and supramarginal gyri. Kenzie et al. used six items from the conventional subtest of the Behavioral Inattention Test (BIT) to examine whether certain lesion sites may be related to either egocentric and allocentric neglect. Among patients with egocentric neglect, star, letter, and line cancellation test performance has been associated with lesion sites in the frontal and parietal lobes. In contrast, among patients with allocentric neglect, line bisection and drawing test performance was associated with lesion sites in the temporal and parietal lobes [28]. These results suggest that investigation into the effects of USN on egocentric and allocentric coordinate systems requires administration of all of the aforementioned desktop tests. 


\section{2) System environment}

In correspondence with the space coordinate system theory described above, this study attempted to develop a system that can project test sheet images on a head-mounted display (HMD). The system environment consisted of an apparatus

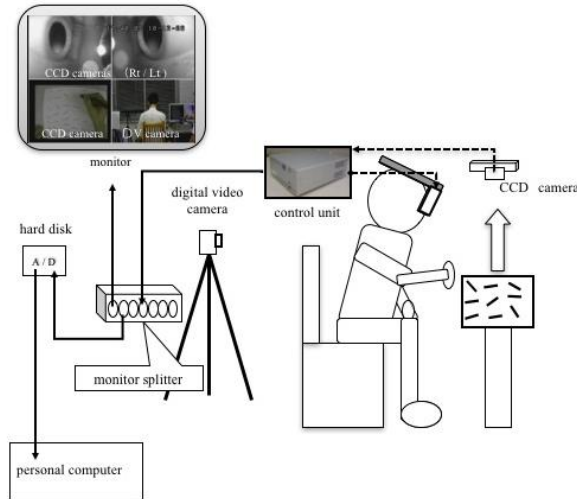

Figure. 2 Experimental apparatus

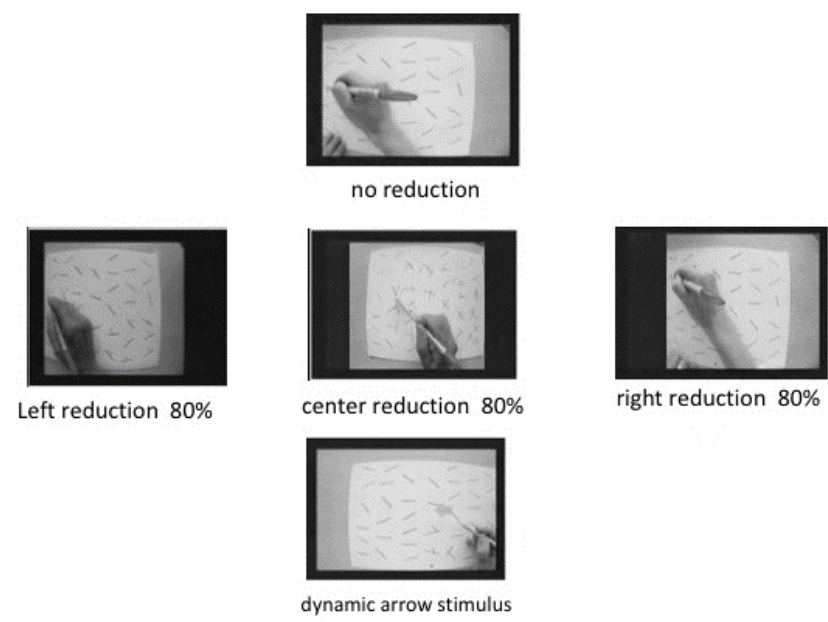

Figure. 3 Visual image conditions by the HMD

for presenting diverse visual information and an apparatus for measuring eye, head, and trunk movements simultaneously (Fig. 2). The HMD was designed to limit the visual field of the subject to a test sheet placed on a desk. A charge-coupled device (CCD) camera affixed to the upper part of the HMD to produce an allocentric condition where the displayed visual image would remain static regardless of head motion. In other words, the system compels the subject to perform visual searches with eye movements.

\section{3) System framework}

The overall system consists of an apparatus for presenting visual information and an apparatus for simultaneously measuring eye, head, and trunk movements. The apparatus for presenting visual information consists of two small CCD cameras secured above a desk, a main control unit for processing and controlling test sheet images captured by the CCD cameras, and an HMD for projecting the processed images to the subject. Each CCD camera captures images for one of the eyes, which are then fed to the left and right screens of the HMD. The resulting binocular disparity enables the reproduction of stereoscopic vision.
The control unit includes a function that allows the examiner to reduce or move images displayed on the HMD.

(a) Visual information presentation (image processing)

Images were processed for the purpose of diversifying the visual information presented to subject. The test sheet image

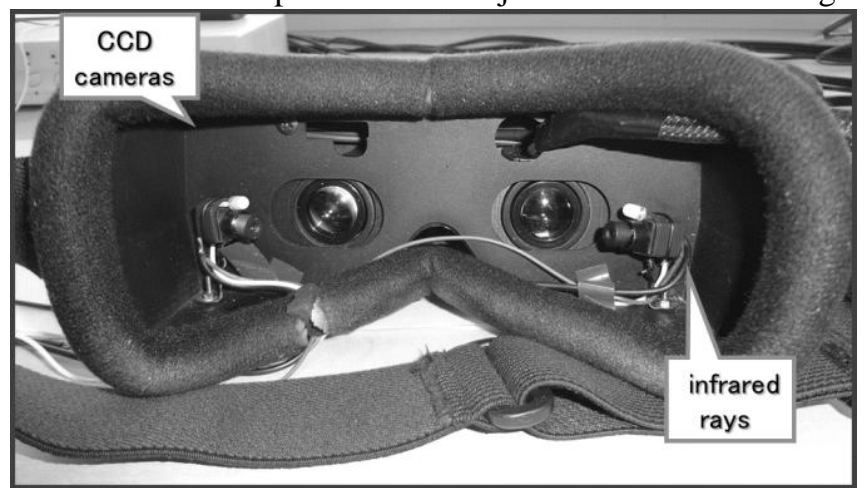

Figure. 4 The inside in the HMD

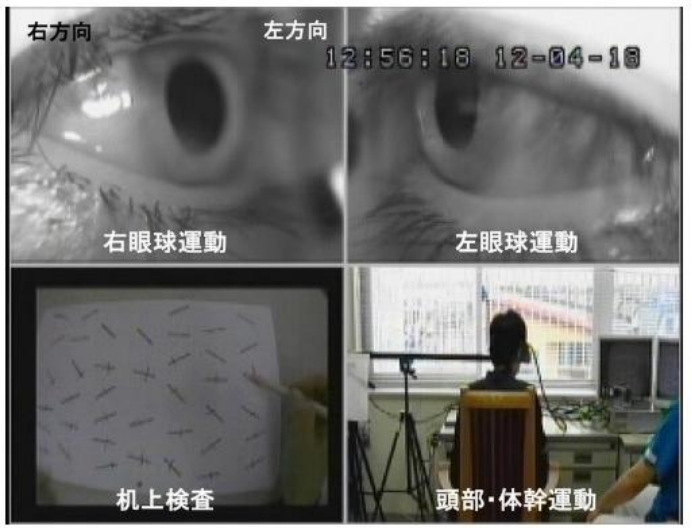

Figure. 5 Video-image in synchronous with eye movement

recorded by the $\mathrm{CCD}$ cameras was reduced according to the ratios listed below and displayed to subjects in the visual field defect (VFD) region or neglected space. In addition, arrows were superimposed onto the test sheet as an attentional mechanism to cue the subject toward the side of visuospatial perception deficit.

The following image reduction conditions were designated for presenting visual information (Fig. 3).

1) No reduction: The image recorded by the CCD camera was projected to the subject at the actual size ( $0 \%$ reduction).

2) Centered reduction: The displayed image was reduced toward the center of the sheet by $80 \%$.

3) Rightward reduction: The image was reduced toward the right side of the sheet by $80 \%$.

4) Leftward reduction: The image was reduced toward the left side of the sheet by $80 \%$.

The attentional mechanism comprised an arrow that traveled leftward from the right edge of the screen under the zero-reduction condition. This was intended as a dynamic stimulus for cuing the attention of the subject toward the neglected side.

(b) Simultaneous measurement of eye, head, and trunk movement

An ultra-compact complementary metal-oxide-semi-conductor (CMOS) camera (MO-S788-4F; Keiyo Techno Co.Ltd., Tokyo.) was installed within the 
HMD to measure eye movement. This NTSC video camera shot only monochrome images, with a minimum illumination of 0.05 lux and horizontal and vertical scan rates of 15.734 $\mathrm{kHz}$ and $60 \mathrm{~Hz}$ respectively, but offered greater sensitivity than comparable models. The dimensions were $8.0 \times 8.0 \mathrm{~mm}$ (width $\times$ height) and the image sensor comprised 250,000

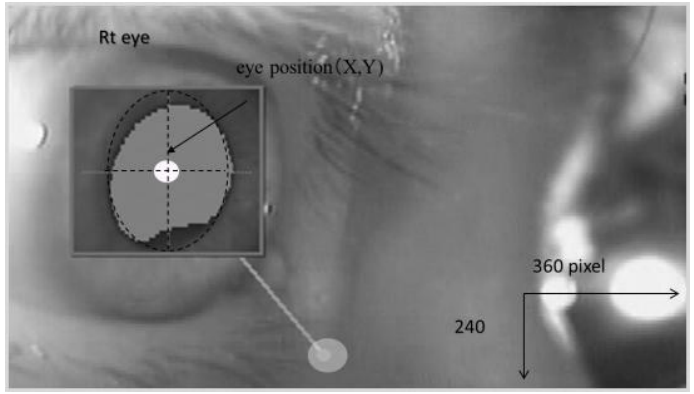

Figure. 6 Eye movement analysis by binarization processing of eye image

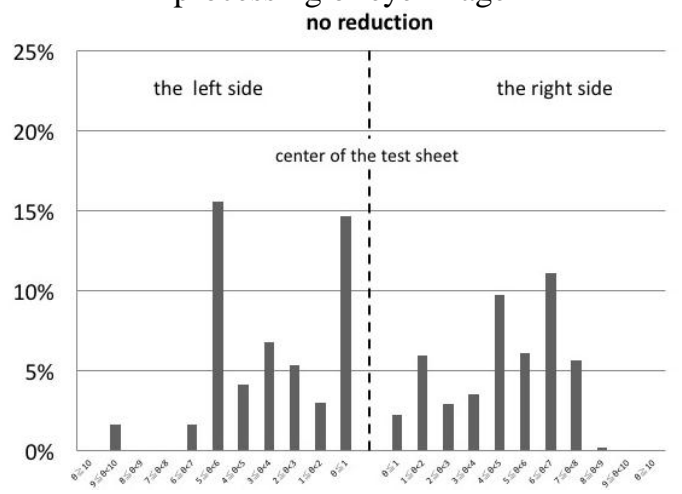

Figure. 7 A sample of histogram of the distribution of eye positions in a healthy subject

valid pixels $(510 \times 492)($ Fig. 4$)$. The examiner manually adjusted two CMOS cameras in the HMD so that each eye was positioned in the center of the monitor. Because the visual field was dark when the HMD was worn by the subject, an ultra-compact infrared light source was used to provide illumination for the camera.

A digital video camera recorded trunk and head movements from the posterior while the subject performed the desktop test. These images plus inputs from both eyes were synchronized with a multi-display adapter, and the resulting 4-quadrant display (Fig. 5) was saved onto a hard disk drive.

\section{B. A novel test method}

1) Desktop test using an HMD to display diverse visual information

A chair sitting position was adopted as the basic measurement posture. First, the test sheet was placed on the desk in alignment with the midline of the body, and the subject was assessed while performing the line cancellation test without the HMD. Next, the HMD was fastened onto the subject and adjustments were made to ensure that the test sheet image was positioned in the center of the liquid crystal display (LCD). The subject performed the test under zero reduction, centered reduction, rightward reduction, leftward reduction, and attention cuing conditions.
2) Eye movement analysis

(a) Eye movement analysis

Recorded eye movements were analyzed using the

Frame-DIAS IV motion analysis application (DKH Co., Ltd., Tokyo.). Using the pupil position obtained from binarization processing of the eye image (Fig. 6), pixel coordinates (X1, Y1) for the center of the eye were established at a sampling Table. 1 Physical and clinical characteristics of patients with visual field defects (VFD) and unilateral spatial neglect (USN) patients.

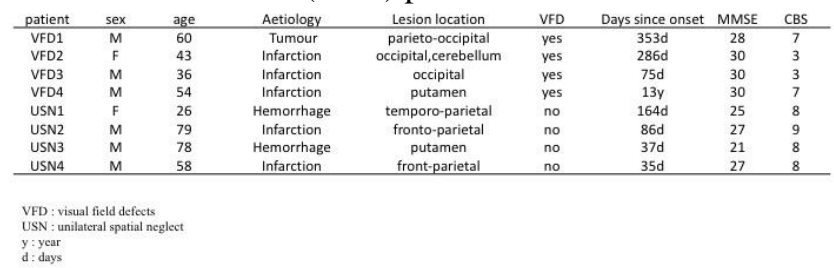

rate of $20 \mathrm{~Hz}$. Observations and recordings were conducted while the eyes were open except blinking.

\section{(b) Eye deviation}

The eye position when the gaze of the subject was aimed at the center of the test sheet was adopted as a reference point for eye deviation. A histogram with bins equal to 1 degree of horizontal eye movement was then constructed to represent the distribution of eye positions during task execution (Fig. 7) and to calculate position ratios for the left and right sides of the test sheet.

\section{Subjects}

A total of eight subjects participated in this study, comprising four with USN and four with VFD (Table 1). All subjects have visual problems of the left side. Among the USN subjects, right hemisphere damage was confirmed through MRI and neglect symptoms through items of the CBS. The CBS has been reported as a valid, reliable assessment tool for USN symptoms in ADL [12]. Each item of the CBS is scored on a 0 to 3 scale of sensitivity, with 0 indicating no neglect and 3 indicating severe neglect. Lower scores on the CBS indicate better functioning with range of 0 point to 30 points. Patients who were unable to maintain a sitting posture in an ordinary chair or wheelchair, those with poor visual acuity, as well as those with severe dementia who were less than 20 points of the Mini-Mental State

Examination score were excluded from this study. Informed consent was obtained from all the subjects prior to their inclusion in the study. This study received the approval of the Ethics Committee of the Graduate School of Information Science and Technology of Hokkaido University (2010-03).

\section{Procedure}

The chair sitting position was adopted as the basic measurement posture. First, a baseline assessment was conducted with the subject performing the line cancellation test without the HMD. Next, measurement was initiated after the HMD was secured to the subjects and the examiner adjusted the CMOS cameras in the HMD. The examiner instructed subjects to fix their gaze on the center of the test sheet and recorded eye images during the calibration for the testing. The following four conditions were applied in the order shown below to VFD and USN patients when subjects 
International Journal of New Technology and Research (IJNTR)

ISSN:2454-4116, Volume-3, Issue-9, September 2017 Pages 14-21

were performing the line cancellation test while wearing the HMD: 1$)$ no reduction ( $0 \%$ reduction) of the test sheet image shot by two small CCD cameras secured above the desk; 2) $80 \%$ reduction of the image toward the center; 3) $80 \%$ reduction of the image toward the right side; and 4) $80 \%$ reduction of the image toward the left.

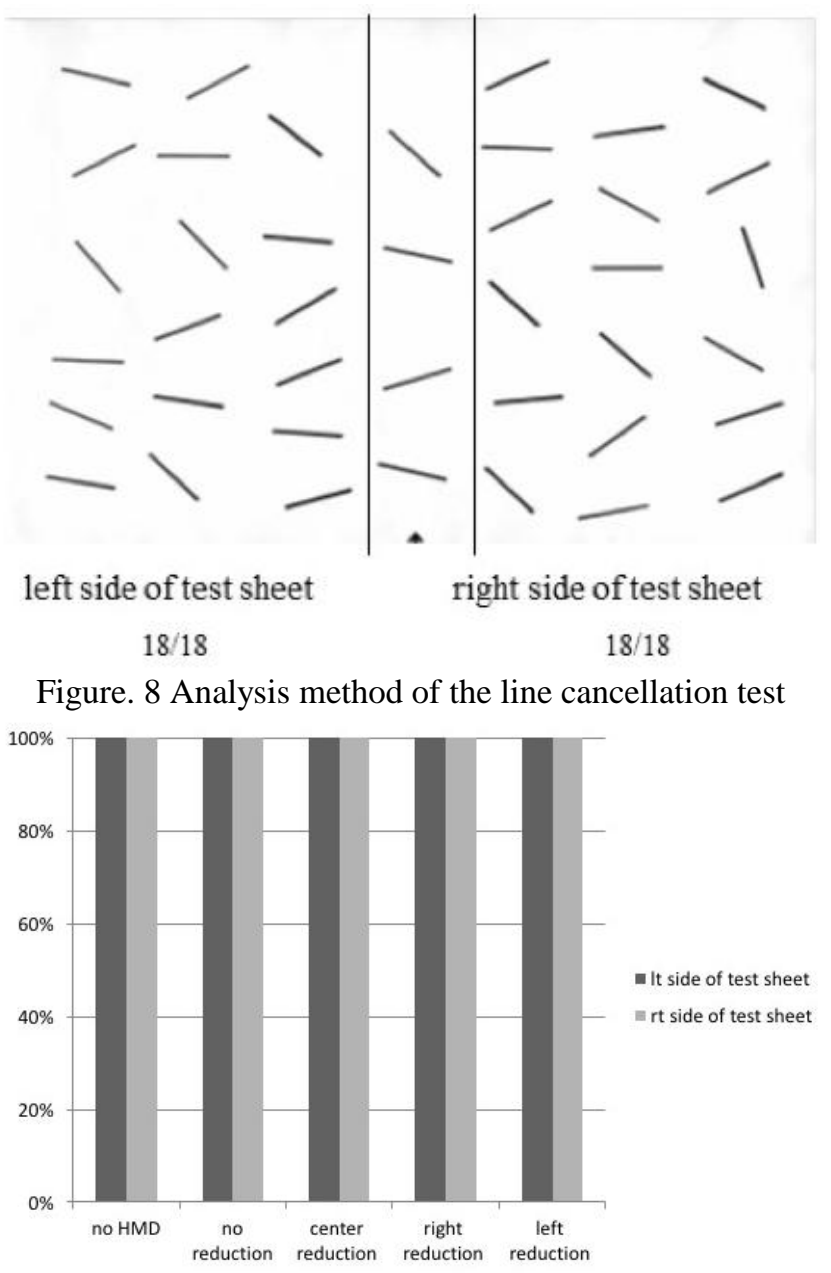

Figure. 9 Percentage of correct answers in line cancellation test for the VFD patient.

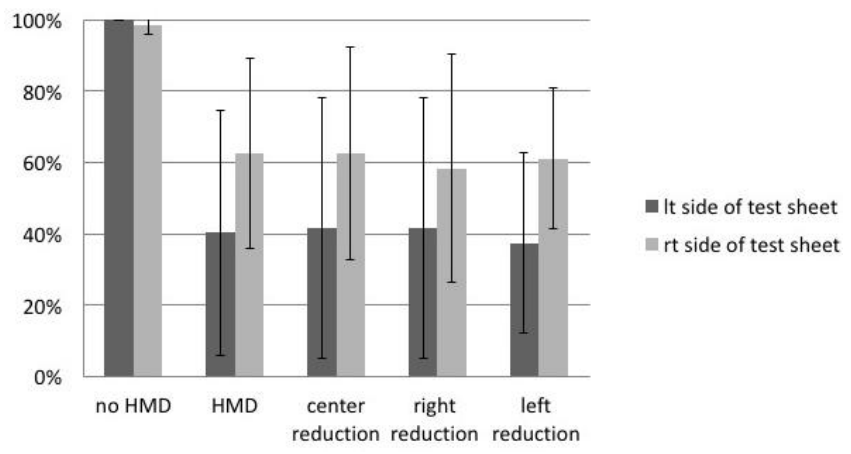

Figure. 10 Percentage of correct answers in line cancellation test for the USN patient.

\section{E. Study Items}

To assess visuospatial perception, the line cancellation test sheet was divided into left and right sides, excluding the four lines in the center row, and correct response rates for both sides were obtained and compared for all conditions (Fig. 8). In terms of eye movement analysis, left and right eye position ratios were calculated using the eye position when the subject gazed at the center of the test sheet as a reference point, as described in II-A-(1). Analysis focused on detecting differences in horizontal eye deviation among the visual presentation conditions during cancellation task execution on either side of the sheet. Collected data were subject to analysis of variance and to multiple comparisons using the Bonferroni method of at the $1 \%$ or $5 \%$ significance level.

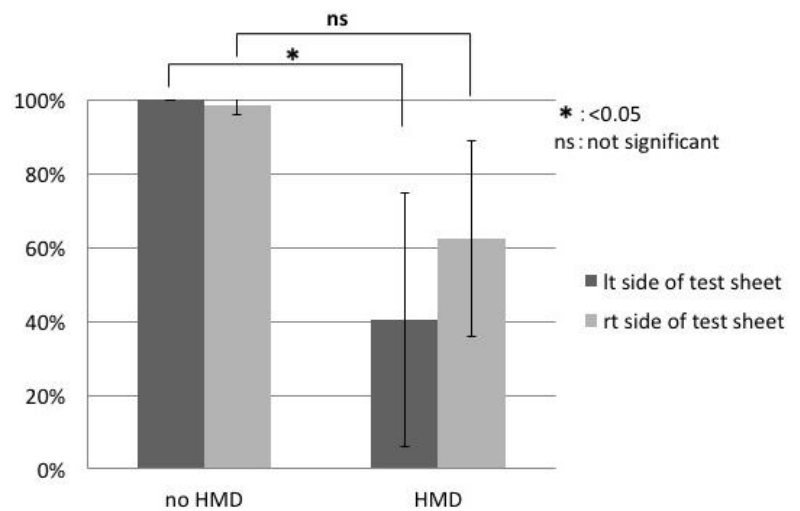

Figure. 11 Percentage of correct answers in line cancellation test with and without the HMD in the USN patient.

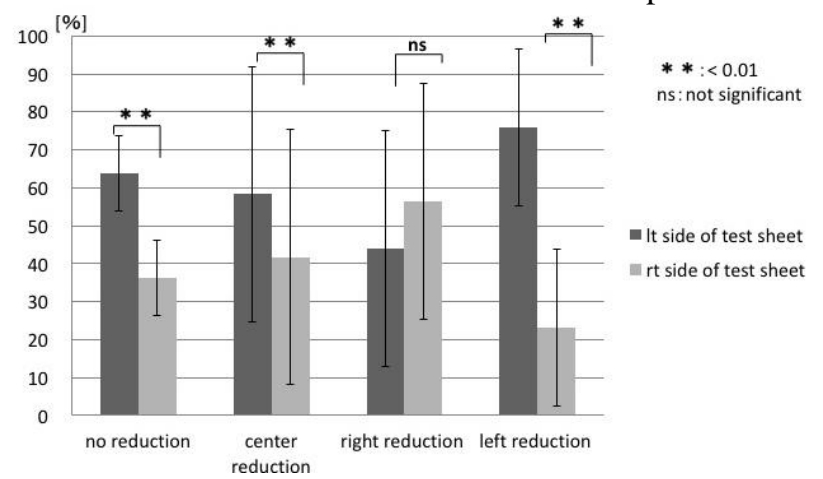

Figure. 12 The rate of eye movement deviation toward the right or left side of the test sheet in the VFD patient.

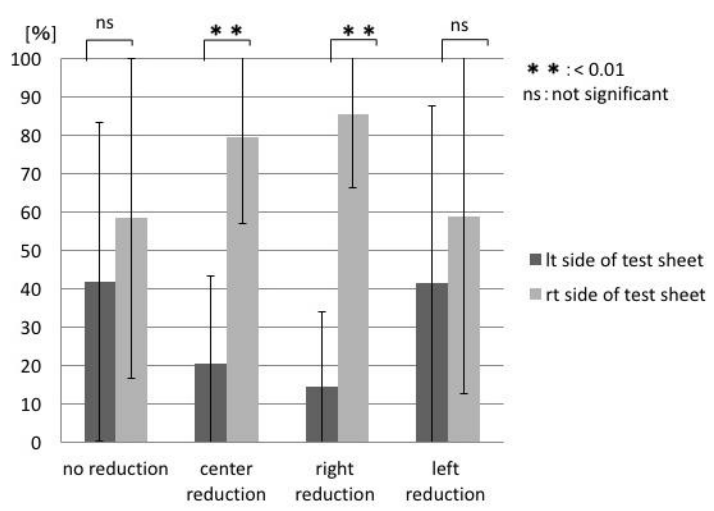

Figure. 13 The rate of eye movement deviation toward the right or left side of the test sheet in the USN patient.

\section{RESULTS}

\section{A. Evaluation of visuospatial perception}

Among VFD patients, no decline in the correct response rate was detected in all conditions for left and right sides of the sheet (Fig. 9).

Among USN patients, the correct response rate for the left side of the sheet was $100 \%$ under non-HMD baseline assessment. With the HMD, the same correct response rate exhibited a decreasing trend with $40 \%$ for zero reduction, 
$42 \%$ for centered and rightward reduction, and $38 \%$ for leftward reduction. For the right side of the sheet, rates were

$98 \%$ for non-HMD baseline assessment, and with the HMD $63 \%$ for zero and centered reduction, $59 \%$ for rightward reduction and $61 \%$ for leftward reduction (Fig. 10). In other words, correct response rates for the left side of the sheet dropped significantly when the HMD was worn (Fig. 11).

Table 2 Motion analysis of eye, a head and a trunk movement for the VFD and USN patients.

\begin{tabular}{|c|c|c|c|c|c|}
\hline & Movement & no reduction & center reduction & right reduction & left reduction \\
\hline VFD1 & $\begin{array}{l}\text { Haed and Trunk } \\
\text { Eve }\end{array}$ & $\mathrm{L}$ & $L$ & $L$ & $\mathrm{~L}$ \\
\hline \multirow{2}{*}{ VFD2 } & Haed and Trunk & $\mathrm{L}$ & $\mathrm{L}$ & - & $\mathrm{L}$ \\
\hline & Eye & $\mathrm{L}$ & L & $\mathrm{L}$ & $\mathrm{L}$ \\
\hline \multirow{2}{*}{ VFD3 } & Haed and Trunk & - & - & - & - \\
\hline & Eye & $\mathrm{L}$ & $\mathrm{R}$ & $\mathrm{R}$ & $\mathrm{L}$ \\
\hline \multirow{2}{*}{ VFD4 } & Haed and Trunk & - & - & - & L \\
\hline & Eye & L & L & $\mathrm{R}$ & $\mathrm{L}$ \\
\hline \multirow{2}{*}{ USN1 } & Haed and Trunk & L & L & L & L \\
\hline & & L & L & $\mathrm{R}$ & L \\
\hline \multirow{2}{*}{ USN2 } & Haed and Trunk & $\mathrm{R}$ & $\mathrm{R}$ & $\mathrm{R}$ & $\mathrm{R}$ \\
\hline & Eye & $\mathrm{R}$ & $\mathrm{R}$ & $\mathrm{R}$ & $\mathrm{R}$ \\
\hline \multirow{2}{*}{ USN3 } & Haed and Trunk & - & - & - & - \\
\hline & Eye & $\mathrm{R}$ & $\mathrm{R}$ & $\mathrm{R}$ & $\mathrm{R}$ \\
\hline \multirow{2}{*}{ USN4 } & Haed and Trunk & - & - & $\mathrm{R}$ & $\mathrm{R}$ \\
\hline & Eye & L & $\mathrm{R}$ & $\mathrm{R}$ & $\mathrm{L}$ \\
\hline
\end{tabular}

\section{B. Eye movement deviation}

Among VFD patients, eye positions were found to deviate toward the left side of the sheet at a higher rate for the zero, centered, and leftward reduction conditions, with a particularly large trend for leftward reduction. Conversely, a higher rate of deviation toward the right side of the sheet was observed for rightward reduction (Fig. 12).

In terms of the ratios for USN patients, eye positions deviated toward the right in all conditions, with a particularly large trend seen in centered and rightward reduction. In addition, ratios were similar between leftward and zero reduction (Fig. 13).

\section{Eye, head, and trunk movement analysis}

VFD patients 1 and 2 showed a tendency for left head and trunk rotation concurrent with eye's leftward movement. VFD patients 3 and 4 mostly displayed no head or trunk movement. USN patient 1 showed a tendency toward left head and trunk rotation. In contrast to VFD patients, USN patient 2 exhibited a tendency for right head and trunk rotation concurrent with eye's rightward movement. Although USN patient 3 exhibited a tendency for eye movement toward the right, no head or trunk movement was observed. For USN patient 4, eye movement was not associated with head and trunk movements (Table 2)."

\section{DISCUSSION}

Among VFD patients, none of the HMD visual field conditions adopted in this study affected line cancellation test performance. However, the correct response rate for the left side of the sheet among USN patients dropped when the HMD conditions were applied.

Since the CBS results of USN patients who participated in this study indicated left neglect in ADL, it is plausible, as previously reported by Tanaka et al. [17-20] that this HMD system featuring diverse visual indexes could discern USN symptoms that otherwise would not have been detected through conventional desktop tests. Furthermore, correct response rates were found to be lower for the right side of the sheet (i.e., the unimpaired side) in VFD and USN patients. Given that all subjects were right-handed, by starting the cancellation task from the right side, subjects may have possibly failed to recognize from the image on the HMD monitor that the right hand was covering lines on the right part of the sheet.

In the word perception study focusing on neglect of the left part of words and letters, Reinhart et al. found that passive head rotation toward the neglected area reduced text omissions on the left, but had no effect on word omissions on the left [29]. Niemeier et al. reported that when USN patients were provided with a frame-like target window for a letter search task, a stronger gradient of attention toward the non-neglected side was observed and they became less likely to turn to the left [30]. In light of the prior studies mentioned above, the act of viewing the test sheet image in the HMD is likely to have restricted attention range and diverted attention toward the direction of non-neglect.

In comparison with the zero-reduction condition, correct response rates for the left side of the sheet (i.e., the impaired side) were higher for centered and rightward reduction and lower for leftward reduction. The higher rates for centered and rightward reduction could be explained by the left side of the sheet moving toward the on-screen area of non-neglect, which did not happen with zero reduction. In contrast, in the case of leftward reduction the left side of the sheet moved toward the on-screen area of neglect, which thereby may have precipitated inferior performance in this condition.

Among VFD patients, eye positions shifted at a higher ratio toward the left side of the sheet (i.e., VFD side) in zero, centered, and leftward reduction, and toward the right side of the sheet (i.e., non-VFD side) in rightward reduction. The stronger VFD-side orientation in the leftward reduction condition could be explained by a larger proportion of the test sheet being displayed on the VFD side, which in turn prompted greater compensatory eye movement in visual searches. Moreover, right-side orientation in the rightward reduction condition could be explained by a larger proportion of the test sheet being displayed on the non-VFD side. On the other hand, eye positions of USN patients deviated toward the non-neglected side under all conditions. This effect was relatively larger in the centered and rightward reduction conditions and smaller in the leftward reduction condition. Karnath et al. examined USN patients who searched for a target object in complete darkness, and discovered that eye movement in visual searches tended to deviate toward the ipsilesional side [31]. Another visual search study employing a line bisection test found that VFD patients were more likely to shift the gaze toward the left edge of the VFD area when bisecting lines, while USN patients deviated gaze to the right side without exploring the left edge during the bisection task [32]. The HMD study presented here also observed that eye positions deviated to the left (i.e., VFD side) in VFD patients and to the right (i.e., non-neglected side) in USN patients 
International Journal of New Technology and Research (IJNTR)

ISSN:2454-4116, Volume-3, Issue-9, September 2017 Pages 14-21

(Fig. 12, 13). The condition is similar to an allocentric coordinate environment where head movement does not change the image position was conceivably achieved by projecting the test sheet shot by the cameras fastened above the desk on the HMD monitor, subsequently making it possible to detect USN.

The results of head and eye motion analysis established that VFD patients tended to rotate the head to the VFD side in the leftward reduction condition. Considering that eye movement velocity in the left direction decreased under the leftward reduction condition, VFD subjects may have tried to compensate to a greater degree with head and trunk activity. VFD patient 2 may have exhibited a tendency to orient the trunk to the direction of deficit due to a limited residual visual field. Acute-phase USN patients have been reported to frequently deviate gaze to the right, with head deviation being observed in severe cases [33, 34]. USN patient 2, who presented with severe neglect, indicated a tendency for right head rotation, suggesting that the environmental circumstances of presenting visual information via the HMD influenced head movement. Nonetheless, USN patient 1 exhibited left head rotation in addition to leftward eye movement under all conditions. The results of this study thus indicate that it is possible to actively cue the attention of chronic patients to the left direction, even under circumstances that is easy to deviate to the right direction. Consequently, the results suggested that the versatile visual condition produced by the HMD may be useful to assess a visual pathology and barriers of USN and VFD patients in relation to their problems of ADL individually. However, the present data of patients were shortage because of the difficulties for collecting the data of the same impairment level of USN or VFD. It is necessary to pile further data to verify our results in near future.

\section{CONCLUSION}

This study performed examinations using a new developed HMD device for presenting diverse visual information and a system for simultaneously measuring eye, head and trunk movements. The HMD system may be available and effective for evaluating and training visual recognition and ADLs in VFD and USN patients as a clinical device. Furthermore, head and eye movement analysis using the system enabled elucidation of differences in visuospatial perception deficits between VFD and USN patients to a certain degree.

\section{CONCLUSION}

A conclusion section is not required. Although a conclusion may review the main points of the paper, do not replicate the abstract as the conclusion. A conclusion might elaborate on the importance of the work or suggest applications and extensions.

\section{APPENDIX}

Appendixes, if needed, appear before the acknowledgment.

\section{ACKNOWLEDGMENT}

The authors thank the subjects for their participation.

\section{REFERENCES}

[1] Zhang X, Kedar S, Lynn MJ, et al.: Natural history of homonymous hemianopia. Neurology, 2006, 66: 901-905.

[2] Shibata K, Nishimura Y, Kondo H, et al.: Isolated homonymous hemianopsia due to lateral posterior choroidal artery region infarction: a case report. Clin Neurol Neurosurg, 2009, 111: 713-716.

[3] Zhang X, Kedar S, Lynn MJ, et al.: Homonymous hemianopia in stroke. J Neuroophthalmol, 2006, 26: 180-183.

[4] George S, Hayes A, Chen C, et al.: The effect of static scanning and mobility training on mobility in people with hemianopia after stroke: a randomized controlled trial comparing standardized versus non-standardized treatment protocols. BMC Neurol, 2011, 11: 87.

[5] Zangemeister WH, Stark L: Active head rotations and eye-head coordination. Ann N Y Acad Sci, 1981, 374: 540-559.

[6] Hirayama K, Sakai S, Yamawaki R, et al.: [Visual search training for a case of homonymous field defect with multiple visual dysfunctions]. No To Shinkei, 2004, 56: 403-413.

[7] Mannan SK, Pambakian AL, Kennard C: Compensatory strategies following visual search training in patients with homonymous hemianopia: an eye movement study. J Neurol, 2010, 257: 1812-1821.

[8] Nijboer T, van de Port I, Schepers V, et al.: Predicting functional outcome after stroke: the influence of neglect on basic activities in daily living. Front Hum Neurosci, 2013, 7: 182

[9] Stone SP, Halligan PW, Greenwood RJ: The incidence of neglect phenomena and related disorders in patients with an acute right or left hemisphere stroke. Age Ageing, 1993, 22: 46-52.

[10] Bowen A, McKenna K, Tallis RC: Reasons for variability in the reported rate of occurrence of unilateral spatial neglect after stroke. Stroke, 1999, 30: 1196-1202.

[11] Halligan PW, Cockburn J, Wilson BA: The behavioural assessment of visual neglect. Neuropsychol Rehabil, 1991, 1:5-32.

[12] Azouvi P, Olivier S, de Montety G, et al.: Behavioral assessment of unilateral neglect: study of the psychometric properties of the Catherine Bergego Scale. Arch Phys Med Rehabil, 2003, 84: 51-57.

[13] Hillis AE, Newhart M, Heidler J, et al.: Anatomy of spatial attention: insights from perfusion imaging and hemispatial neglect in acute stroke. J Neurosci, 2005, 25: 3161-3167.

[14] Hillis AE: Rehabilitation of unilateral spatial neglect: new insights from magnetic resonance perfusion imaging. Arch Phys Med Rehabil, 2006, 87: S43-S49.

[15] Lee JH, Ku J, Cho W: A virtual reality system for the assessment and rehabilitation of the activities of daily living. Cyberpsychol Behav 2003,6(4):383-388.

[16] Prothero JD: The treatment of akinesia using virtual images. In Master's thesis Industrial Engineering, University of Washington; 1993.

[17] Tanaka T, Sugihara S, Nara H, et al.: A preliminary study of clinical assessment of left unilateral spatial neglect using a head mounted display system (HMD) in rehabilitation engineering technology. $\mathbf{J}$ Neuroeng Rehabil, 2005, 2: 31

[18] Tanaka T, Ifukube T, Sugihara S, et al.: A case study of new assessment and training of unilateral spatial neglect in stroke patients: effect of visual image transformation and visual stimulation by using a Head Mounted Display system (HMD). J Neuroeng Rehabil, 2010, 7: 20.

[19] Sugihara S, Miyasaka T, Tanaka T: A study of assessment of unilateral spatial neglect using a system for motion analysis of eyes and a head-neck. IEEE/SICE Int Symp Syst Integr, 2009, 67-70.

[20] Sugihara S, Tanaka T, Miyasaka T, et al.: Assessment of visual space recognition in patients with visual field defects using head mounted display (HMD) system: Case study with severe visual field defect. In Proceedings of the Annual International Conference of the IEEE Engineering in Medicine and Biology Society, EMBS, 2013, 6929-6932.

[21] Ishiai S: What do eye-fixation patterns tell us about unilateral spatial neglect? Restor Neurol Neurosci, 2006, 24:261-271.

[22] Fruhmann-Berger M, Karnath HO: Spontaneous eye and head position in patients with spatial neglect. J Neurol, 2005, 252: 1194-1200.

[23] Fruhmann Berger M, Pross RD, Ilg U, et al.: Deviation of eyes and head in acute cerebral stroke. BMC Neurol, 2006, 6: 23

[24] J. T. Kleinman, M. Newhart, C. Davis, J. Heidler-Gary, R. F. Gottesman, and A. E. Hillis, "Right hemispatial neglect: frequency and characterization following acute left hemisphere stroke.," Brain Cogn., vol. 64, no. 1, pp. 50-9, Jun. 2007. 
[25] A. E. Hillis and A. Caramazza, "Deficit to stimulus-centered, letter shape representations in a case of "unilateral neglect,", Neuropsychologia, vol. 29, no. 12, pp. 1223-1240, Dec. 1991.

[26] N. Beschin, R. Cubelli, S. Della Sala, and L. Spinazzola, "Left of what? The role of egocentric coordinates in neglect.," J. Neurol. Neurosurg. Psychiatry, vol. 63, no. 4, pp. 483-9, Oct. 1997.

[27] M. Niemeier and H.-O. Karnath, "Simulating and testing visual exploration in spatial neglect based on a new model for cortical coordinate transformation," Experimental Brain Research, vol. 145, no. 4. pp. 512-519, Aug-2002.

[28] J. M. Kenzie, K. A. Girgulis, J. A. Semrau, S. E. Findlater, J. A. Desai, and S. P. Dukelow, "Lesion Sites Associated with Allocentric and Egocentric Visuospatial Neglect in Acute Stroke.," Brain Connect., vol. 5, no. 7, pp. 413-22, Sep. 2015.

[29] S. Reinhart, I. Keller, and G. Kerkhoff, "Effects of head rotation on space- and word-based reading errors in spatial neglect," Neuropsychologia, vol. 48, no. 13, pp. 3706-3714, 2010.

[30] M. Niemeier and H. O. Karnath, "Stimulus-driven and voluntary saccades are coded in different coordinate systems.," Curr. Biol., vol. 13, no. 7, pp. 585-589, 2003.

[31] H. O. Karnath, M. Fetter, and J. Dichgans, "Ocular exploration of space as a function of neck proprioceptive and vestibular input--observations in normal subjects and patients with spatial neglect after parietal lesions.," Exp. Brain Res., vol. 109, no. 2, pp. 333-42, May 1996.

[32] J. Barton, "Ocular search during line bisection. The effects of hemi-neglect and hemianopia," Brain, vol. 121, no. 6, pp. 1117-1131, Jun. 1998.

[33] M. Fruhmann-Berger and H.-O. Karnath, "Spontaneous eye and head position in patients with spatial neglect.," J. Neurol., vol. 252, no. 10 , pp. 1194-200, Oct. 2005.

[34] E. Becker and H.-O. Karnath, "Neuroimaging of eye position reveals spatial neglect.," Brain, vol. 133, no. Pt 3, pp. 909-14, Mar. 2010.

Toshiaki Tanaka is a Professor at The University of Tokyo and Hokkaido University of Science. He received his $\mathrm{PhD}$ from Hokkaido University, in 1998. His research of interest includes Kinesiology, Rehabilitation Science, Ergonomics, and Assistive Engineering. He is a Board of Director, The Japanese Society for Wellbeing Science and Assistive Technology. He is author of a book, Science of Balance Training for Preventing a Falling (2013) and a coauthor of a book, Eye Movement Input Device Designed to Help Maintain Amyotrophic Lateral Sclerosis (ALS) Patients (2012). He has published over 170 research papers in his research of interest.

Shunichi Sugihara is a Manager at Department of Rehabilitation, Sapporo Shuyukai Hospital. He received his PhD from Hokkaido University, in 2015. His research of interest includes Neuroscience, Rehabilitation Science, and Assistive Engineering.

Norio Kato is a Lecturer at The University of Tokyo and Hokkaido University of Science. He received his PhD from Hokkaido University, in 2016. His research of interest includes Virtual Reality, IoT, Kinesiology, Rehabilitation Science and Assistive Engineering. 